\title{
Temperature-Dependent Network Modules of Soil Methanogenic Bacterial and Archaeal Communities
}

OPEN ACCESS

Edited by: Graeme W. Nicol, Université de Lyon, France

Reviewed by:

Heli Juottonen,

University of Jyväskylä, Finland

Oliver Schmidt,

University of Bayreuth, Germany

*Correspondence:

Pengfei Liu

pengfei.liu@mpi-marburg.mpg.de;

liupfskygre@gmail.com

Specialty section:

This article was submitted to Terrestrial Microbiology, a section of the journal

Frontiers in Microbiology

Received: 11 October 2018

Accepted: 26 February 2019

Published: 12 March 2019

Citation:

Liu P, Klose M and Conrad R

(2019) Temperature-Dependent

Network Modules of Soil

Methanogenic Bacterial and Archaeal

Communities.

Front. Microbiol. 10:496

doi: 10.3389/fmicb.2019.00496

\section{Pengfei Liu*, Melanie Klose and Ralf Conrad}

Max Planck Institute for Terrestrial Microbiology, Marburg, Germany

Temperature is an important factor regulating the production of the greenhouse gas $\mathrm{CH}_{4}$. Structure and function of the methanogenic microbial communities are often drastically different upon incubation at $45^{\circ} \mathrm{C}$ versus $25^{\circ} \mathrm{C}$ or $35^{\circ} \mathrm{C}$, but are also different in different soils. However, the extent of taxonomic redundancy within each functional group and the existence of different temperature-dependent microbial community network modules are unknown. Therefore, we investigated paddy soils from Italy and the Philippines and a desert soil from Utah (United States), which all expressed $\mathrm{CH}_{4}$ production upon flooding and exhibited structural and functional differences upon incubation at three different temperatures. We continued incubation of the pre-incubated soils (Liu et al., 2018) by changing the temperature in a factorial manner. We determined composition, abundance and function of the methanogenic archaeal and bacterial communities using HiSeq Illumina sequencing, qPCR and analysis of activity and stable isotope fractionation, respectively. Heatmap analysis of operational taxonomic units (OTU) from the different incubations gave detailed insights into the community structures and their putative functions. Network analysis showed that the microbial communities in the different soils were all organized within modules distinct for the three incubation temperatures. The diversity of Bacteria and Archaea was always lower at $45^{\circ} \mathrm{C}$ than at 25 or $35^{\circ} \mathrm{C}$. A shift from $45^{\circ} \mathrm{C}$ to lower temperatures did not recover archaeal diversity, but nevertheless resulted in the establishment of structures and functions that were largely typical for soil at moderate temperatures. At 25 and $35^{\circ} \mathrm{C}$ and after shifting to one of these temperatures, $\mathrm{CH}_{4}$ was always produced by a combination of acetoclastic and hydrogenotrophic methanogenesis being consistent with the presence of acetoclastic (Methanosarcinaceae, Methanotrichaceae) and hydrogenotrophic (Methanobacteriales, Methanocellales, Methanosarcinaceae) methanogens. At $45^{\circ} \mathrm{C}$, however, or after shifting from moderate temperatures to $45^{\circ} \mathrm{C}$, only the Philippines soil maintained such combination, while the other soils were devoid of acetoclastic methanogens and consumed acetate instead by syntrophic acetate oxidation coupled to hydrogenotrophic methanogenesis. Syntrophic acetate oxidation was apparently achieved by Thermoanaerobacteraceae, which were especially abundant in Italian paddy soil and Utah desert soil when incubated at $45^{\circ} \mathrm{C}$. Other bacterial taxa were also differently abundant at $45^{\circ} \mathrm{C}$ versus moderate temperatures, as seen by the formation of specific network modules. However, the archaeal OTUs with putative function in 
acetoclastic or hydrogenotrophic methanogenesis as well as the bacterial OTUs were usually not identical across the different soils and incubation conditions, and if they were, they suggested the existence of mesophilic and thermophilic ecotypes within the same OTUs. Overall, methanogenic function was determined by the bacterial and/or archaeal community structures, which in turn were to quite some extent determined by the incubation temperature, albeit largely individually in each soil. There was quite some functional redundancy as seen by different taxonomic community structures in the different soils and at the different temperatures.

Keywords: temperature shifts, methanogenesis pathway, methanogenic community, paddy soil, desert soil, functional redundancy

\section{INTRODUCTION}

Methane production is achieved by anaerobic degradation of organic matter involving a complex microbial community consisting of Bacteria and Archaea. Bacteria (and perhaps some Archaea) hydrolyze polymers (e.g., cellulose); ferment the monomers to short chain fatty acids, alcohols, $\mathrm{CO}_{2}$ and $\mathrm{H}_{2}$, or ferment them to acetate as sole product; and convert the primary fermentation products to acetate, $\mathrm{H}_{2}$ and $\mathrm{CO}_{2}$. Methanogenic archaea then convert either acetate (acetoclastic methanogenesis) or $\mathrm{H}_{2} / \mathrm{CO}_{2}$ (hydrogenotrophic methanogenesis) to $\mathrm{CH}_{4}$ (Zinder, 1993; Schink and Stams, 2013). Methane can also be produced from methyl groups such as methanol, trimethylamine, dimethylsulfide etc., if these compounds occur in the environment (Zinder, 1993). In anoxic soils, $\mathrm{CH}_{4}$ is usually produced by hydrogenotrophic and acetoclastic methanogenesis. The two pathways can be distinguished, since $\mathrm{CH}_{4}$ produced from $\mathrm{CO}_{2}$ reduction is much more depleted in ${ }^{13} \mathrm{C}$ than that from acetate cleavage (Whiticar, 1999; Conrad, 2005). Soils usually contain a rather high diversity of Archaea, including methanogens such as the potentially acetoclastic families Methanosarcinaceae and Methanotrichaceae ['Methanosaetaceae'(Oren, 2014)] as well as the hydrogenotrophic orders Methanocellales, Methanomicrobiales, and Methanobacteriales (Conrad, 2007). The complexity of Bacteria is even larger (Asakawa and Kimura, 2008; Rui et al., 2009; Wegner and Liesack, 2016). Both domains (Archaea and Bacteria) include a large number of taxa of which isolates are lacking and their potential physiology is little known or completely unknown. The extent of taxonomic redundancy within each functional group (e.g., acetoclastic methanogenesis) is also not well known. Such redundancy may be promoted by differentiation in response to environmental factors other than metabolic resources (Louca et al., 2018). Temperature is such an environmental factor and thus, we used it in the present study to affect microbial function and taxonomic structure.

Temperature is a universal regulator of microbial activity (Wiegel, 1990), and also greatly affects $\mathrm{CH}_{4}$ production (YvonDurocher et al., 2014). Microbial community responses to increasing temperatures can be quite complex (Roussel et al., 2015). Temperature not only regulates each individual process by affecting the underlying thermodynamics and kinetics, but also affects population dynamics and the structuring of the entire microbial community (Conrad, 2008). Rice field soils, for example, are able to produce $\mathrm{CH}_{4}$ over a wide temperature range up to about $55^{\circ} \mathrm{C}$ (Yao and Conrad, 2000; Fey et al., 2001). However, the structure and function of the methanogenic microbial community was found to change drastically within a temperature interval of about $42-46^{\circ} \mathrm{C}$ (Conrad et al., 2009). Whereas a mesophilic methanogenic community exists below this interval, above it is replaced by a thermophilic one. This change often coincides with a change in the methanogenic pathway from a mixture of acetoclastic plus hydrogenotrophic to exclusively hydrogenotrophic methanogenesis, and by the replacement of acetoclastic methanogenesis by syntrophic acetate oxidation that is coupled to hydrogenotrophic methanogenesis (Conrad et al., 2009; Liu and Conrad, 2010; Rui et al., 2011). Recently, metatranscriptomics and co-occurrence network analysis revealed that the microbial community of Italian rice field soil consisted of modules characterized by the functional activities of polymer hydrolysis, syntrophic oxidation of key intermediates, and methanogenesis (Peng et al., 2018). Both mesophilic $\left(30^{\circ} \mathrm{C}\right)$ and moderate thermophilic $\left(45^{\circ} \mathrm{C}\right)$ temperatures had a differential effect on all the functional activities (Peng et al., 2018).

In a previous study, we found different methanogenic pathways, depending on whether the soils did or did not contain thermophilic acetoclastic methanogens (Liu et al., 2018). In fact, many different thermophilic methanogens exist in anoxic soils from various regions (Wu et al., 2006), and only few soils have so far been tested with respect to temperature effects on methanogenic microbial communities (Conrad et al., 2009; Rui et al., 2011; Lu et al., 2015; Liu et al., 2018). Therefore, we asked whether the methanogenic microbial communities in soil will always be organized in cooccurrence network modules, which are distinct for different temperatures. Consequently, the microcosms of the three soils incubated for $\sim 250$ days and analyzed in our previous study (Liu et al., 2018) were now considered as a pre-incubation in this study, where their incubation temperature was changed in a factorial manner. They were incubated for a further 3050 days and investigated for the composition of bacterial and archaeal operational taxonomic units (OTUs) of the resulting communities. The temperature shifts were applied to evaluate to which extent the microbial communities were able to respond by changing composition, in particular to see to which extent 
a mesophilic community would regenerate after exposure to elevated temperatures and vice versa.

The diversity of the methanogenic archaeal and bacterial communities seems to be lower under thermophilic than mesophilic conditions. Nevertheless, mesophilic communities and functions can be recovered from thermophilic ones when the temperature is decreased below the temperature interval of about $42-46^{\circ} \mathrm{C}$ (Conrad et al., 2009; Noll et al., 2010), especially if the soil is inoculated with a mesophilic community. Structure and function of the methanogenic communities apparently change in parallel. However, changes in communities have so far only been recorded by molecular fingerprint analysis, which addresses the composition of the communities only coarsely. This is especially true for bacterial communities (Noll et al., 2010). With the availability of deep sequencing techniques (e.g., Illumina) such methodological limitations are now released. Therefore, and because we recently discovered quite different structures of mesophilic and thermophilic methanogenic communities in different soils (Liu et al., 2018), we decided to investigate how these structures and their functions change when incubation temperatures are shifted between mesophilic and thermophilic conditions.

The overall objectives of our study were to unravel the taxonomic redundancy of functional microbial groups, in particular syntrophic acetate oxidation and acetoclastic and hydrogenotrophic methanogenesis, after exposure to mesophilic $\left(25\right.$ and $\left.35^{\circ} \mathrm{C}\right)$ and thermophilic $\left(45^{\circ} \mathrm{C}\right)$ temperatures, and to find out whether these communities were organized in co-occurrence networks. We first hypothesized that function (pathway of $\mathrm{CH}_{4}$ production in particular) at different temperatures would be determined by the existence or non-existence of specific functional microbial groups. We secondly hypothesized that these functional groups may form different taxonomic community structures depending on soil and temperature. Thirdly, we asked whether taxonomic diversity and putative functional redundancy are affected by temperature and by shifts between these temperatures.

We used two different paddy soils (from Italy and from the Philippines) and one desert soil (from Utah, United States), which all produced $\mathrm{CH}_{4}$ in anoxic soil slurries. After preincubation at mesophilic $\left(25\right.$ and $\left.35^{\circ} \mathrm{C}\right)$ or thermophilic $\left(45^{\circ} \mathrm{C}\right)$ conditions, temperatures were changed in a factorial manner. The methanogenic pathways were determined by stable isotope fractionation and the microbial community structures by the taxonomic composition and abundance of Bacteria and Archaea.

\section{MATERIALS AND METHODS}

\section{Soil Sampling, Soil Incubation and Chemical Analyses}

The same soils from three different climate zones were used as in our previous study (Liu et al., 2018), including two paddy soils from Italian Rice Research Institute in Vercelli (collected in 2013) and the International Rice Research Institute (IRRI) in Los Baños, Philippines (collected in 2012), respectively, and one desert soil from a natural site in Utah, United States (collected in 2009).
The physicochemical properties of each soil were measured by standard methods (Fu et al., 2018). The same batch of desert soil had been used in a previous study (Angel et al., 2012). The incubations were set up in 2015 and continued into the year 2016.

Anoxic soil incubations were separated into two phases. During pre-incubation, soil slurries were prepared by mixing $50 \mathrm{~g}$ dry soil with $50 \mathrm{ml}$ of deionized, sterile, anoxic water and $100 \mathrm{mg}$ cellulose in $150-\mathrm{ml}$ bottles. The bottles were sealed with rubber stoppers and flushed with $\mathrm{N}_{2}$, and then incubated at three different temperatures (each with three replicates): 25 , 35 , and $45^{\circ} \mathrm{C}$. Treatments were designated as I25, I35, and I45 for Italian paddy soil, P25, P35, and P45 for the Philippines paddy soil and U25, U35, and U45 for Utah soil, respectively (Supplementary Figure S1). After about 250 days incubation, aliquots of soil slurries were collected and stored frozen at $-20^{\circ} \mathrm{C}$ for later molecular analysis. Part of the data from the preincubation have been reported in our previous paper (Liu et al., 2018 ), i.e., production rates and isotopic compositions of $\mathrm{CO}_{2}$ and $\mathrm{CH}_{4}$; relative abundance of the major bacterial and archaeal phyla; copy numbers of bacterial and archaeal 16S rRNA genes; concentrations of volatile fatty acid. If required for comparison, they are included in the present manuscript as mentioned in the legends of tables and figures. However, the composition of the microbial communities in terms of OTUs has not yet been reported and is subject of the present study.

The remaining soil slurries from the three triplicates at the same temperature were mixed and dispensed into $26-\mathrm{mL}$ tubes, with $10 \mathrm{~g}$ slurry per tube. The soil slurries were kept stirred by a high speed magnetic mixer to avoid particles settling during the dispensing of preincubated soil. In addition, the soil slurries were amended with $10 \mathrm{mg}$ of cellulose as additional carbon source. The tubes were closed with black rubber stoppers, flushed with $\mathrm{N}_{2}$, pressurized to 0.5 bar overpressure. The soil slurries were either continuously incubated under the same temperature as during pre-incubation or switched to one of the other two temperatures (Supplementary Figure S1). In total, nine treatments (each with three replicates) were established for each soil. For Italian paddy soil, treatments were designated as $\mathrm{I} 25 \mathrm{e} 25, \mathrm{I} 25 \mathrm{e} 35, \mathrm{I} 25 \mathrm{e} 45$, and $\mathrm{I} 35 \mathrm{e} 25, \mathrm{I} 35 \mathrm{e} 35$, $\mathrm{I} 35 \mathrm{e} 45$, and $\mathrm{I} 45 \mathrm{e} 25, \mathrm{I} 45 \mathrm{e} 35, \mathrm{I} 45 \mathrm{e} 45$. For the Philippines paddy soil, treatments were designated as $\mathrm{P} 25 \mathrm{e} 25, \mathrm{P} 25 \mathrm{e} 35, \mathrm{P} 25 \mathrm{e} 45$, and P35e25, P35e 35, P35e45, and P45e25, P45e35, P45e45. For Utah desert soil, treatments were designated as U25e25, $\mathrm{U} 25 \mathrm{e} 35, \mathrm{U} 25 \mathrm{e} 45$, and U35e25, U35e $35, \mathrm{U} 35 \mathrm{e} 45$, and U45e25, U45e35, U45e45 (Supplementary Figure S1). Soil slurries were incubated at the respective temperatures for 30-50 days. During the incubation, accumulation of $\mathrm{CH}_{4}$ and $\mathrm{CO}_{2}$ in the headspace was measured using a gas chromatography (GC) equipped with methanizer and flame ionization detector as previously described (Yao et al., 1999). The $\delta^{13} \mathrm{C}$ of $\mathrm{CH}_{4}$ and $\mathrm{CO}_{2}$ was measured by GC combustion isotope ratio mass spectrometry (GC-CIRMS) (Conrad et al., 2012). The apparent isotopic fractionation factor for conversion of $\mathrm{CO}_{2}$ to $\mathrm{CH}_{4}$ was determined by $\alpha_{\text {app }}=\left(\delta^{13} \mathrm{CO}_{2}+10^{3}\right) /\left(\delta^{13} \mathrm{CH}_{4}+10^{3}\right)$ (Fey et al., 2004). Volatile fatty acids were measured by high-performance liquid chromatography (HPLC) (Liu and Conrad, 2010). Soil slurries of each treatment were collected at the end of incubation and stored 
frozen at $-20^{\circ} \mathrm{C}$ for later analysis of volatile fatty acids and for molecular analysis.

\section{DNA Extraction, qPCR, Illumina Library Preparation and Sequencing}

The same protocol was used for DNA extraction and quantification of archaeal and bacterial 16S rRNA genes by qPCR as in our previous study (Liu et al., 2018).

The Illumina libraries were prepared as in our previous study (Liu et al., 2018) using primers 515F and 806R targeting the V4 region of the $16 \mathrm{~S}$ rRNA gene (approximately 250 nucleotides) for both Archaea and Bacteria (Bates et al., 2011). Library 1 included all samples from the end of the pre-incubation (Liu et al., 2018). Libraries 2-4 included samples of Italian, the Philippines and Utah soils, respectively, at the end of incubation at different temperatures. All libraries were sequenced on an ILLUMINA HISEQ 2000 system using $2 \times 250$ cycle combination mode by Max Planck-Genome-Centre (Cologne, Germany). Sequence data sets were stored in the same project as our previous study (Liu et al., 2018) with accession number SRP133538.

\section{Bioinformatic Analyses}

Similar to our previous study (Liu et al., 2018), sequence processing was mainly based on the UPARSE pipeline (Edgar, 2013) and additionally included tools from the programs QIIME 1.9.0 (Caporaso et al., 2010), CUTADAPT 1.9.1 (Martin, 2011), and USEARCH v8.0.1623 (Edgar, 2010). In brief, the adaptor sequence ahead of the barcoded forward primer and reverse primers (Supplementary Table S1) were trimmed with CUTADAPT. Un-trimmed sequences were discarded. Pairedend reads were then merged and demultiplexed using UPARSE python scripts ${ }^{1}$. After quality control, de novo chimera filtering and singleton filtering, species level OTUs for 16S rRNA genes were obtained at $97 \%$ sequence identity. Taxonomic identities of 16S rRNA gene OTUs were assigned with the Ribosomal Database Project (RDP) Classifier against the SILVA 128 SSU Ref database (Pruesse et al., 2007) at a confidence level of $80 \%$ (Wang et al., 2007). OTUs belonging to Bacteria and Archaea were separated according to their taxonomic identities. For alpha-diversity analyses, the OTU tables with singletons were subsampled using the rrarefy function of the vegan package (version 2.4-4) (Oksanen et al., 2007) within $\mathrm{R}^{2}$ based on the lowest number of sequences available from each samples, i.e., 281947 for bacterial- and 10162 for archaeal16S rRNA genes. This procedure standardizes the measures needed for comparison. Rarefied OTU tables were then processed within packages vegan, entropart (version 1.4-7) and phyloseq (version 1.19.1) (McMurdie and Holmes, 2013) in R. BrayCurtis distance matrix was calculated based on OTUs tables without singletons, non-metric multidimensional scaling analysis (NMDS) was then carried out using the ordination function within R package phyloseq (McMurdie and Holmes, 2013). Differences in population structure between treatments within

\footnotetext{
${ }^{1}$ https://drive5.com/python/

${ }^{2}$ http://www.r-project.org/
}

each types of soils were analyzed using the statistic ANOSIM (based on Bray-Curtis dissimilarities, permutations $=999$ ) in R.

Heatmaps were constructed with the pheatmap (pretty heatmaps version 1.0.8) and vegan packages using OTUs that explained most of the differences between samples. Such OTUs were identified by principal components analysis (PCA) of the Hellinger transformed data using the prcomp function in vegan. Twenty five archaeal OTUs and 50 bacterial OTUs explaining most of the differences between samples were defined as the OTUs contributing the largest absolute loadings in the first and second dimensions of the PCA (Hernandez et al., 2017), obtained from the rotation output file. To construct heatmaps for Archaea, a total of 35,39 , and 32 unique OTUs were obtained for Italian, the Philippines and Utah soil, respectively (since some of the OTUs were selected from more than one PCA axes). To construct heatmaps for Bacteria, a total of 88,86 , and 85 unique OTUs were obtained for bacterial $16 \mathrm{~S}$ rRNA genes for Italian, the Philippines and Utah soil, respectively. The OTU abundances were converted to percentage of sequences from each sample, and Hellinger distances were calculated as described above. Hierarchical clustering of the distance matrix was carried out with the "complete" method using hclust function.

\section{Co-occurrence Network Analysis}

Three co-occurrence networks based on the abundance of $16 \mathrm{~S}$ rRNA gene OTUs (including both bacterial and archaeal OTUs) were constructed for Italian, the Philippines and Utah soils, respectively, by following the pipelines developed by Williams et al. (2014). Generally, the OTU table without singletons was first rarefied to the smallest sample depth for all three soils (here 281947 reads for bacterial 16S rRNA gene and 10162 reads for archaeal 16S rRNA gene). OTUs with less than $0.1 \%$ or $1 \%$ maximum relative abundances for Bacteria and Archaea, respectively, were filtered out. OTUs from Bacteria and Archaea were combined and Spearman's rank correlations between selected OTUs were calculated based on absolute reads abundance. Pairs with Spearman's correlation coefficient $0.8 \leq \rho \leq 0.9$ and FDR corrected $p$-value $<0.01$ were used for co-occurrence network construction. Networks were constructed with the robust correlations as weighted edges using Gephi software ${ }^{3}$. The network layout was generated using the force-based algorithm Fruchterman-Reingold (Fruchterman and Reingold, 1991). Modules within network were computed using the multilevel modularity optimization algorithm (Blondel et al., 2008).

\section{Statistical Analyses}

For qPCR data, the means \pm standard deviation (SD) from all replicates were calculated. Gene copy numbers were logtransformed to satisfy the normality assumptions, and the analysis of variance was performed to test significant differences between treatments using the DUNCAN test within agricolae package (version 1.2-8) (Mendiburu and Simon, 2015) in R. For the $\alpha$ diversity index, analysis of variance was performed to test

${ }^{3}$ https://gephi.github.io/ 
significant differences between treatments using the KruskalWallis test within agricolae package.

\section{RESULTS}

\section{Functional Process Data}

Patterns of $\mathrm{CH}_{4}$ production (Figure 1) and $\mathrm{CO}_{2}$ production (Supplementary Figure S2) were dependent on the temperature during pre-incubation and were different at 25, 35, and $45^{\circ} \mathrm{C}$ (Figure 1). Production rates of $\mathrm{CO}_{2}$ tended to increase when the temperature was increased after pre-incubation and vice versa (Supplementary Figure S2). Also $\mathrm{CH}_{4}$ production increased when shifting from $25^{\circ} \mathrm{C}$ during pre-incubation to $35^{\circ} \mathrm{C}$ (Figure 1). Shifting to $45^{\circ} \mathrm{C}$ also increased $\mathrm{CH}_{4}$ production in the paddy soils (Italy, the Philippines), but decreased them in the Utah desert soil (Figure 1). Shifting from 35 to $25^{\circ} \mathrm{C}$ generally resulted in a lag phase of several days

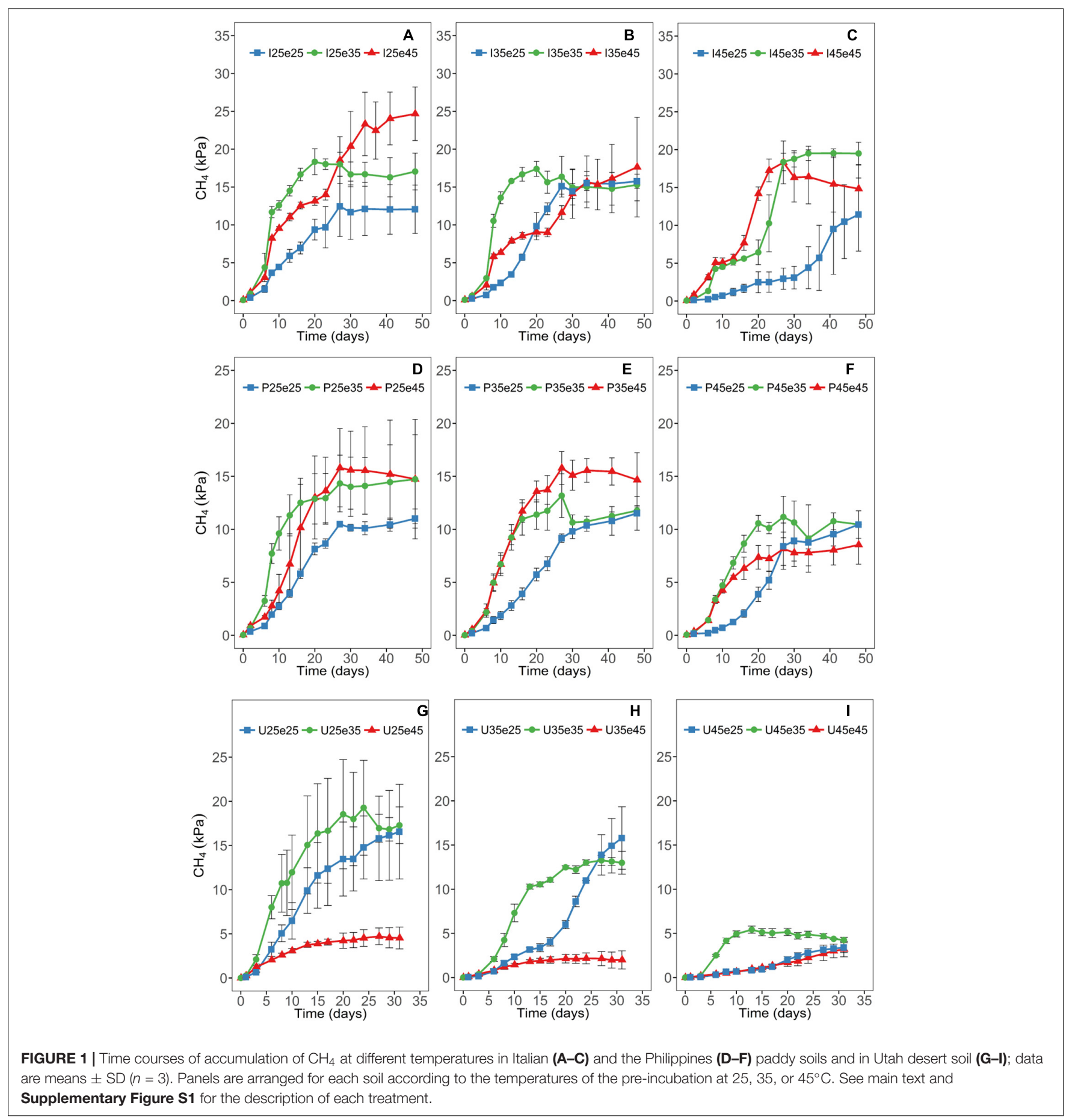


and in decreased $\mathrm{CH}_{4}$ production (Figure 1). Shifting from $45^{\circ} \mathrm{C}$ during pre-incubation to lower temperatures resulted in similar rates at $35^{\circ} \mathrm{C}$, but significantly decreased the rates at $25^{\circ} \mathrm{C}$ (Figure 1). However, this pattern was only observed in the two paddy soils (Italy, the Philippines), while in the
Utah desert soil $\mathrm{CH}_{4}$ production at 25 and $45^{\circ} \mathrm{C}$ became very low (Figure 1).

High values of $\alpha_{\text {app }}$ (about 1.08) are characteristic for $\mathrm{CH}_{4}$ production exclusively from $\mathrm{CO}_{2}$ and low values (about 1.04) of $\alpha_{\text {app }}$ are characteristic for $\mathrm{CH}_{4}$ production from both $\mathrm{CO}_{2}$ and

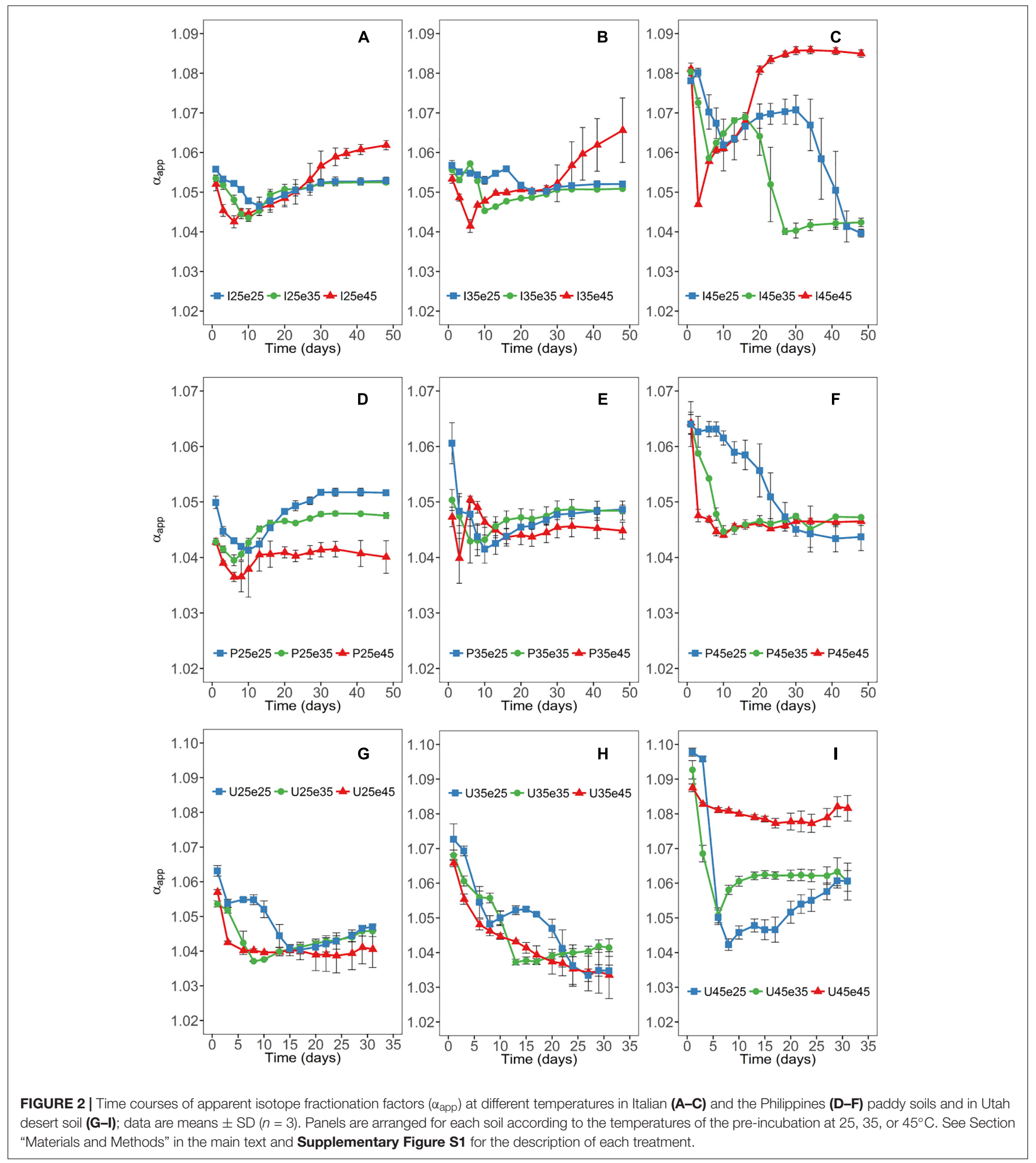


acetate (Fey et al., 2004). Continuous incubation at $45^{\circ} \mathrm{C}$ resulted in the establishment of high $\alpha_{\text {app }}$ values in Italian paddy soil and Utah desert soil indicating that $\mathrm{CH}_{4}$ was mainly (or exclusively) produced from $\mathrm{H}_{2} / \mathrm{CO}_{2}$ (Figure 2). In the Philippines paddy soil, by contrast, $\alpha_{\text {app }}$ values eventually became relatively low indicating that $\mathrm{CH}_{4}$ was produced by both acetoclastic and hydrogenotrophic methanogenesis (Figure 2). This happened in the Philippines soil at all temperatures irrespectively of pre-incubation (Figure 2). Relatively low $\alpha_{\text {app }}$ values also established in all the other soils at 25 and $35^{\circ} \mathrm{C}$ irrespectively of the temperature of pre-incubation. However, increase of the pre-incubation temperature of Italian paddy soil (but not of Utah desert soil) to $45^{\circ} \mathrm{C}$ resulted in an increasing trend of

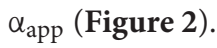

We also determined the concentrations of volatile fatty acids (VFA) at the end of incubation (Supplementary Table S2). In the paddy soils, acetate, propionate and formate were detected, the concentrations being below $1 \mathrm{mM}$. Capronate was only detected in $\mathrm{I} 25 \mathrm{e} 45$. In the Utah desert soil, however, also lactate, butyrate, valerate, iso-valerate, and capronate were frequently detected (Supplementary Table S2). Concentrations of acetate were in Utah desert soil especially high $(2-6 \mathrm{mM})$, and were exceptionally high if temperature was decreased from $45^{\circ} \mathrm{C}$ during preincubation to $25^{\circ} \mathrm{C}$ or $35^{\circ} \mathrm{C}$ (reaching $>70 \mathrm{mM}$ ) (Supplementary Table S2). The accumulation of VFA is consistent with the very low rates of $\mathrm{CH}_{4}$ production after pre-incubation of Utah soil at $45^{\circ} \mathrm{C}$ (Figure 1). The $\mathrm{pH}$ values were generally around neutrality.
However, in Utah soil after pre-incubation at $45^{\circ} \mathrm{C}$ values were between $\mathrm{pH} 6.0$ and 6.5 .

\section{Quantification of Archaeal and Bacterial 16S rRNA Genes}

Copy numbers of archaeal and bacterial 16S rRNA genes were quantified to assess the absolute abundances (gene copies) of Archaea and Bacteria (Figure 3). Copy numbers of archaeal 16S rRNA genes were generally between $1 \times 10^{8}$ and $5 \times 10^{9}$ copies per gram dry soil across all the samples. Copy numbers of bacterial 16S rRNA genes were nearly one order of magnitude higher than archaeal 16S rRNA genes in Italian and Utah soil and they were similar in the Philippines soil (Figure 3). Copy numbers of both archaeal and bacterial 16S rRNA genes were usually slightly higher (often significantly, $p<0.05$ ) in samples incubated at moderate temperatures than at $45^{\circ} \mathrm{C}$. In Utah desert soil, copy numbers of archaeal genes stayed relatively low once soil had been incubated at $45^{\circ} \mathrm{C}$. Another interesting feature was that the copy numbers (both Bacteria and Archaea) increased from the end of pre-incubation to the end of the subsequent incubation indicating microbial growth (Figure 3).

\section{Overall Microbial Diversity and Composition of Microbial Communities}

The composition of the microbial communities in the different treatments of the three soils was assessed by HiSeq sequencing,
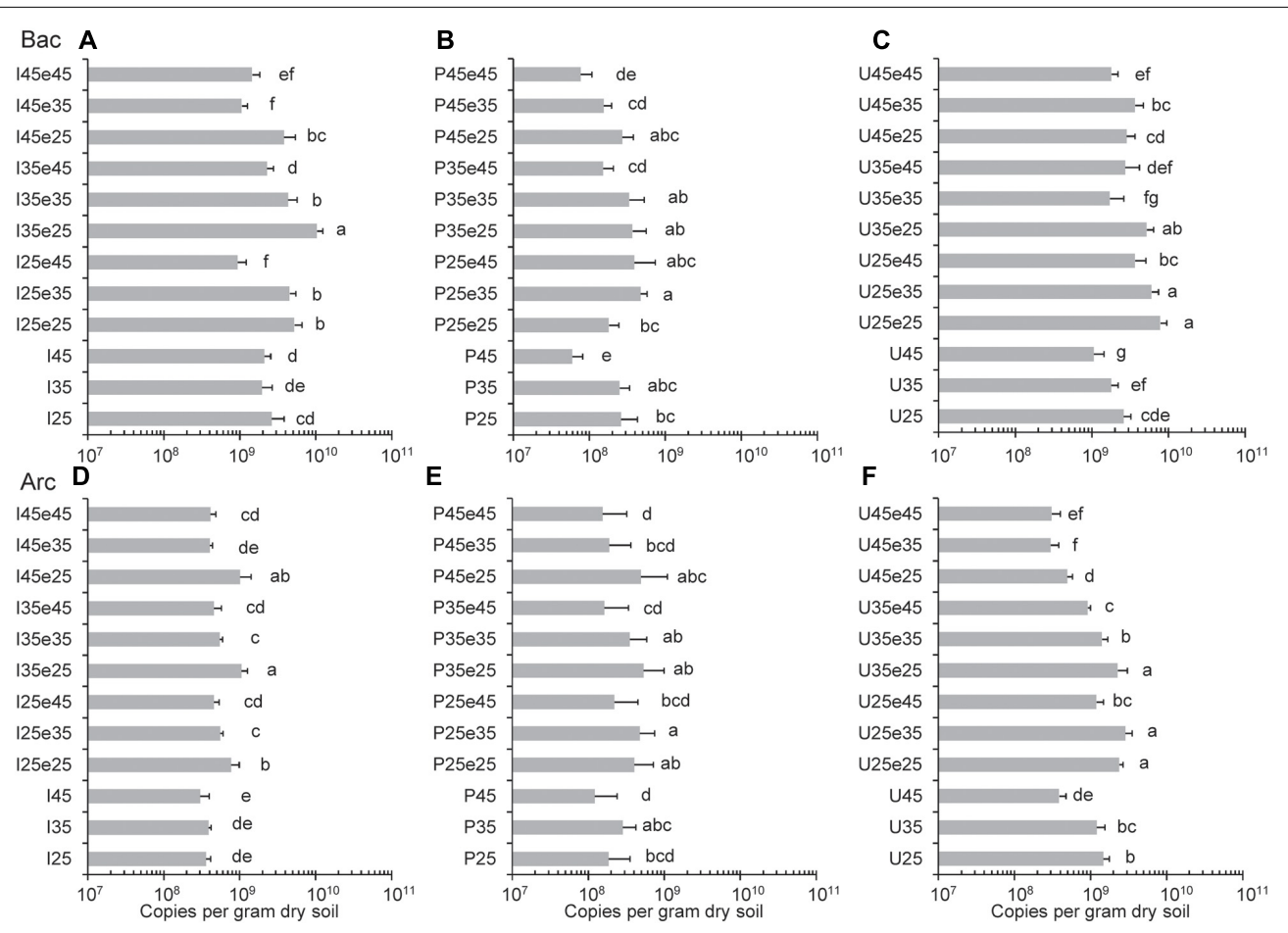

FIGURE 3 | Copy numbers of (A,B,C) Bacterial and (D,E,F) Archaeal 16S rRNA genes in (A,D) Italian paddy soil, (B,E) the Philippines paddy soil and (C,F) Utah desert soil; data are means $\pm \mathrm{SD}$, with $n=3$. Different letters next to the data indicate significant difference $(p<0.05)$ between copy numbers. See Section "Materials and Methods" in the main text and Supplementary Figure S1 for the description of each treatment. Data from the pre-incubation (I25, 35, 45, P25, 35, 45, and U25, 35, 45) were reported in our previous publication (Liu et al., 2018). 
resulting in an average of 12-80 thousands and 300-500 thousands reads for Archaea and Bacteria, respectively (Supplementary Tables S3, S4). The average numbers of OTUs obtained by similarity-based clustering of the sequences (3\% sequence dissimilarity and without singletons) were about 40-200 and 2500-9200 for Archaea and Bacteria, respectively. Current sequencing depth covered almost the entire archaeal and bacterial diversity in all three soils, although some rare taxa were probably missed (Supplementary Tables S3, S4). For both Archaea and Bacteria, pre-incubation at $45^{\circ} \mathrm{C}$ or increase of the temperature to $45^{\circ} \mathrm{C}$ often significantly decreased diversity as described by species richness (Chaol estimators), Shannon and Fisher indices and evenness (J). The decrease was most pronounced in Italian paddy soil and Utah desert soil, but was less obvious in the Philippines paddy soil (as exemplified by the Shannon index in Figure 4). By contrast, decrease of the temperature from $45^{\circ} \mathrm{C}$ during pre-incubation to $25^{\circ} \mathrm{C}$ or $35^{\circ} \mathrm{C}$ did not result in significant increase of diversity. Apparently, diversity was not recovered once it had been lost.

At the end of the pre-incubation at 25,35 , and $45^{\circ} \mathrm{C}$ the archaeal communities in the different soils were different, and again changed with continuing incubation (Supplementary Figure S3). The Italian paddy soil at 25 and $35^{\circ} \mathrm{C}$ mainly contained Methanobacteriales, Methanocellales, Methanosarcinaceae, and Methanotrichaceae, but also some Methanomicrobiales. In addition there were Bathyarchaeota, Soil Crenarchaeota Group (SCG), Woesarchaeota and also some Thermoplasmatales (Supplementary Figure S3A). This composition was rather well maintained upon continued incubation at the same temperature or upon shift to another moderate temperature. In the Philippines paddy soil, the archaeal communities were qualitatively similar at all the different temperatures (Supplementary Figure S3B). Notably, the community always consisted of all the major archaeal groups, including Methanobacteriales, Methanocellales, Methanomicrobiales, Methanosarcinaceae, Methanotrichaceae, Bathyarchaeota, SCG, Woesarchaeota, and Thermoplasmatales (Supplementary Figure S3B). Hence, acetoclastic Methanosarcinaceae and Methanotrichaceae were always present. The archaeal community in Utah desert soil rarely contained acetoclastic Methanotrichaceae, and did not contain Woesearchaeota (Supplementary Figure S3C). At $45^{\circ} \mathrm{C}$ Utah desert soil also did not contain any acetoclastic Methanosarcinaceae, and did not contain Bathyarchaeota. Further incubation at $45^{\circ} \mathrm{C}$ resulted in increase of Methanobacteriales ( $80 \%)$ while Methanocellales almost disappeared (Supplementary Figure S3C).

The bacterial communities consisted of 18 different taxonomic classes with $>2 \%$ relative abundance (Supplementary Figure S4). Pre-incubation at $45^{\circ} \mathrm{C}$ generally resulted in higher relative abundance of Clostridia and other Firmicutes than pre-incubation at moderate temperatures (Supplementary Figure S4). Decrease of temperature did not reverse this pattern. In addition to the general responses of bacterial compositions, we also investigated the changes of putative syntrophic bacteria including Thermoanaerobacteraceae, Peptococcaceae, Syntrophomonadaceae, and Helicobacteraceae

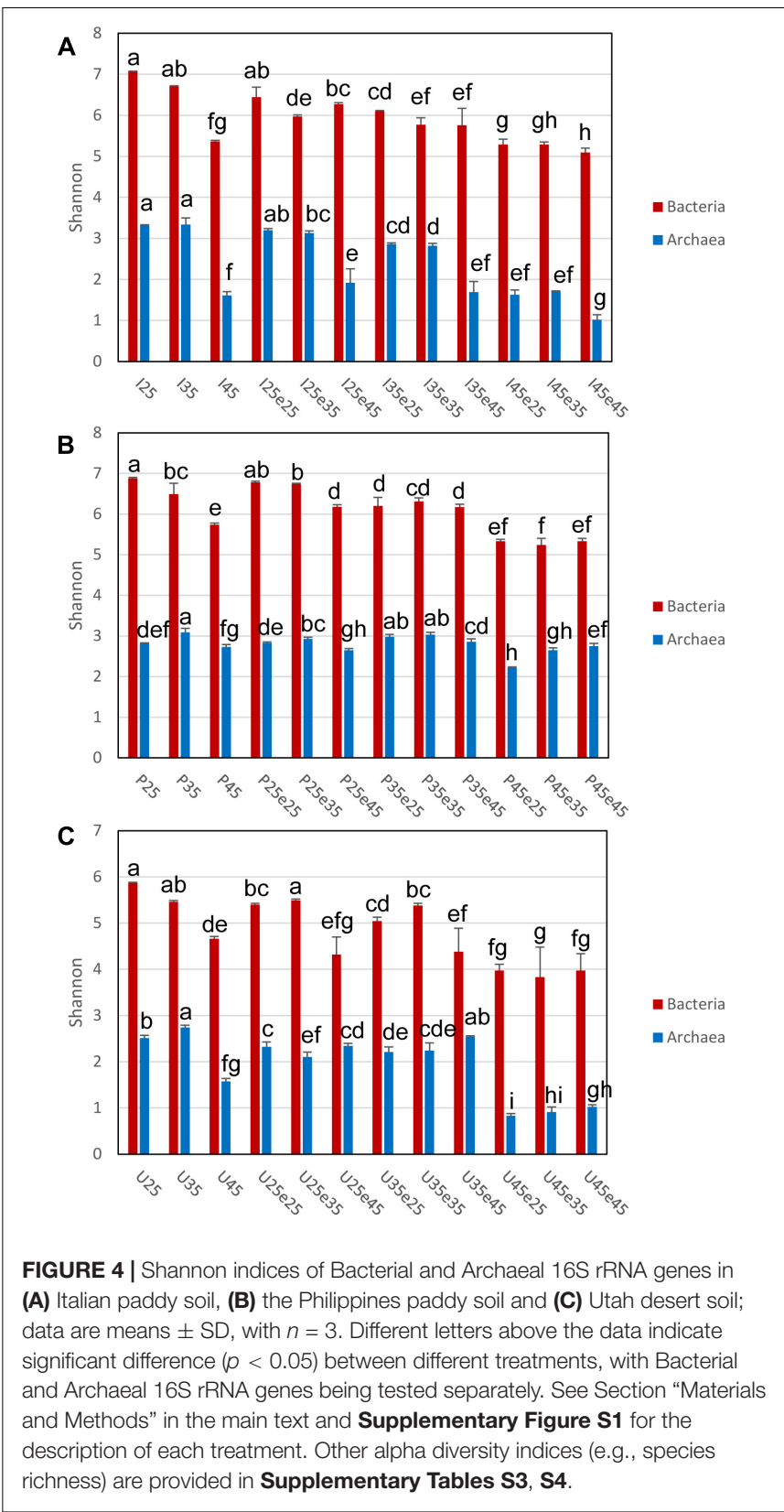

(all belonging to the phylum Clostridia), as well as Syntrophobacteraceae, Syntrophaceae, and Geobacteraceae (all belonging to the class Delta-Proteobacteria) (Supplementary Figure S5). In particular, Thermoanaerobacteraceae were interesting, since they represent putative acetate oxidizing syntrophs. In Italian soil, this group always showed a relatively high abundance, when the soil was incubated at $45^{\circ} \mathrm{C}$ (Supplementary Figure S5A), while in the Philippines soil, the abundance of Thermoanaerobacteraceae was generally low (Supplementary Figure S5B).

Non-metric multidimensional scaling (NMDS) analysis of archaeal OTUs showed differences in community compositions between all the different treatments and for all the three soils 
(Supplementary Figure S6), and many of these differences were significant (see $R$ - and $P$-values in Supplementary Figure S6). For Italian soil, the I45e45 treatment formed a unique archaeal cluster. Other samples incubated at $45^{\circ} \mathrm{C}$ either during pre-incubation or after temperature change clustered together and were separated from samples that were exclusively incubated at moderate temperatures (Supplementary Figure S6A). For Utah and the Philippines soils, samples were mainly separated by the incubation temperature during pre-incubation (Supplementary Figures S6B,C).

Non-metric multidimensional scaling also confirmed that the bacterial communities within each soil were significantly different for the different treatments (see $R$-and $P$-values in Supplementary Figure S7). Similar with Archaea, the Bacteria in the samples were mainly separated by the incubation temperature during pre-incubation, and there was little effect by the temperature change, except when temperature was increased to $45^{\circ} \mathrm{C}$ (Supplementary Figure S7).

\section{Heatmap Analysis of Microbial Operational Taxonomic Units}

In order to identify the most important OTUs, 25 archaeal and 50 bacterial OTUs with the highest contributions in defining the first two PCA axes were selected and used to create a heatmap for each soil and for Archaea (Figure 5) and Bacteria (Figures 68), respectively. The heatmaps showed distinct clusters of the archaeal and bacterial community compositions. The clustering of samples in the heatmaps was similar to that of NMDS analysis (Supplementary Figures S6, S7) and differentiated the samples largely according to the temperatures used for incubation. In the Italian soil (Figures $\mathbf{5 A}, \mathbf{6}$ ), four main clusters (from right to left) separated treatments with (1) pre-incubation at either 25 or $35^{\circ} \mathrm{C}$ followed by incubation at $45^{\circ} \mathrm{C}$; (2) preincubation at $45^{\circ} \mathrm{C}$ followed by incubation at the same or other temperatures; (3) pre-incubation at $25^{\circ} \mathrm{C}$ followed by incubation at either 25 or $35^{\circ} \mathrm{C}$; and (4) pre-incubation at $35^{\circ} \mathrm{C}$ followed by incubation at either 25 or $35^{\circ} \mathrm{C}$. In the Philippines and Utah soils (Figures 5B,C, 7, 8), there were three main clusters, which showed a similar separation as in the Italian soil, with the first cluster containing the treatments with pre-incubation at $45^{\circ} \mathrm{C}$, while the other two clusters contained the treatments with preincubations at either 25 or $35^{\circ} \mathrm{C}$ including the treatments that were later shifted to $45^{\circ} \mathrm{C}$.

\section{Network Analysis of Microbial Operational Taxonomic Units}

The combined archaeal and bacterial OTUs of each soil were used for network analysis. Each soil exhibited three major modules (Supplementary Figures S8-S10). Most of the OTUs enriched in the cluster with pre-incubation at $45^{\circ} \mathrm{C}$ of the archaeal and bacterial heatmaps (shown in red in Figures 5-8) were found in one of the three major modules $\left(45^{\circ} \mathrm{C}\right)$ of the networks (Supplementary Figures S8-S10). The OTUs enriched in clusters with pre-incubation at 25 and $35^{\circ} \mathrm{C}$ (shown in green and blue, respectively, in Figures 5-8) also formed two network modules (see also Supplementary Datasets 1-3 for

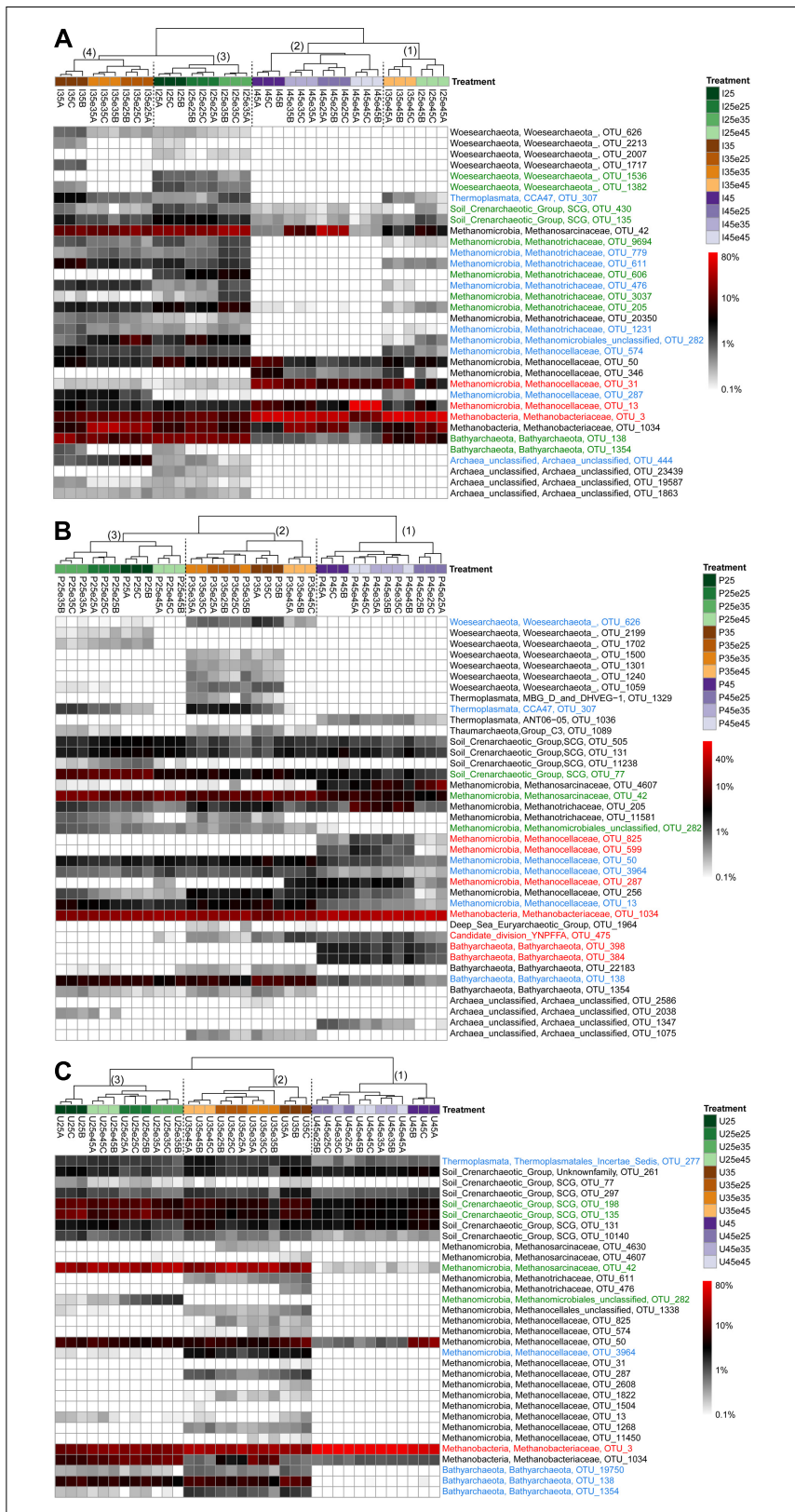

FIGURE 5 | Relative abundances of selected archaeal OTUs that explain most of the differences between treatments in (A) Italian paddy soil, (B) the Philippines paddy soils, and (C) Utah desert soil. Descriptions of OTUs selection, sample clustering and heatmap construction are given in Section "Materials and Methods" in the main text. See Section "Materials and Methods" in the main text and Supplementary Figure $\mathbf{S 1}$ for the description of each treatment. The color scale is logarithmic to emphasize rare taxa, with $0.1 \%$ being set as the lower bound for logarithmic transformation. Numbers above the dendrogram indicate clusters described in the main text. Names of the OTUs were colored according to the three major modules in the Supplementary Figures S8-S10, i.e., green, blue, and red corresponding to module 25,35 , and $45^{\circ} \mathrm{C}$, respectively (see Supplementary Figures S8-S10). OTUs in black were not in the network.

details), but each of these modules contained OTUs from both 25 and $35^{\circ} \mathrm{C}$ (Supplementary Figures S8-S10). Hence these 


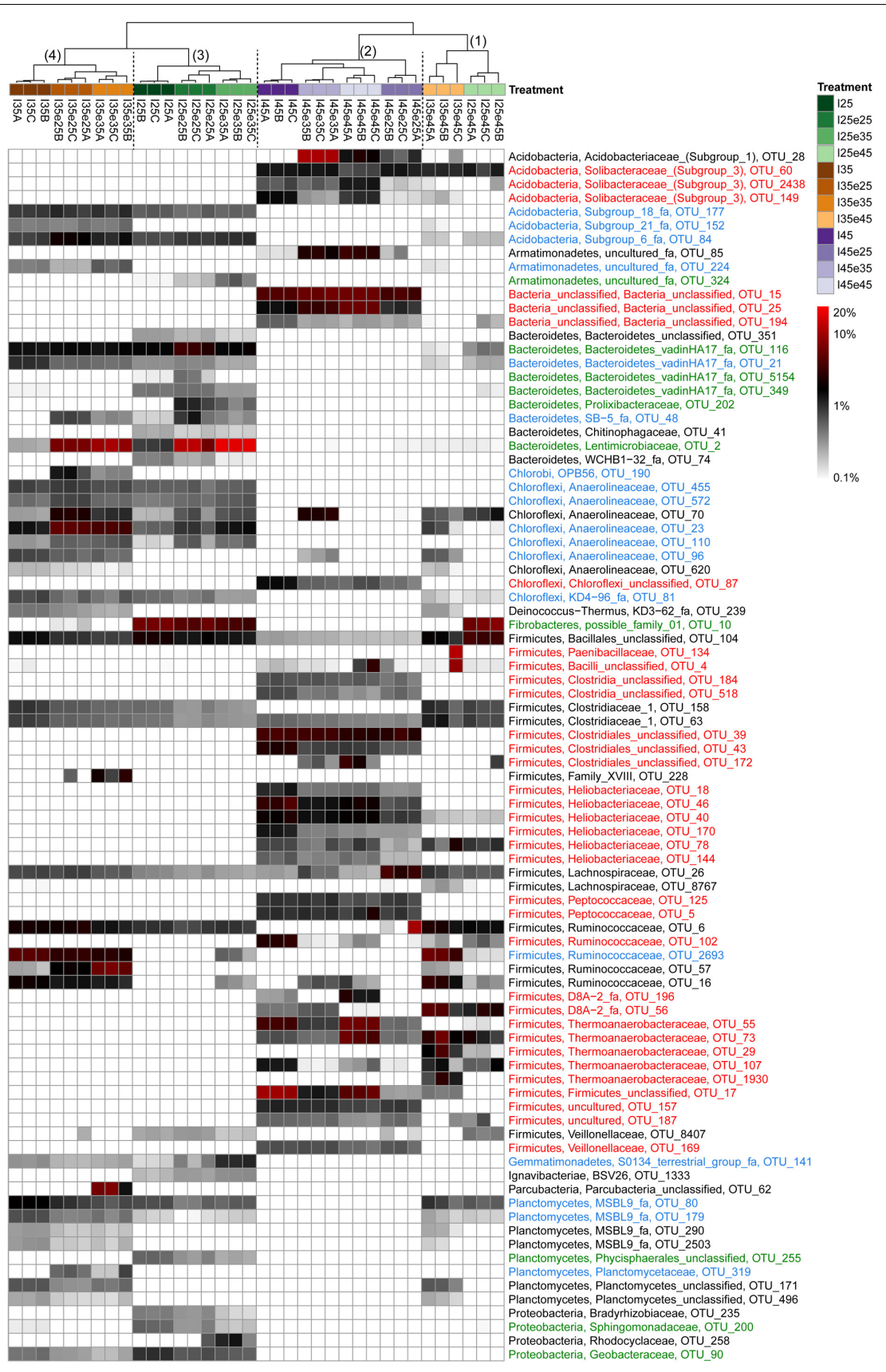

FIGURE 6 | Relative abundances of selected bacterial OTUs that explain most of the differences between treatments in Italian paddy soil. Descriptions of OTUs selection, sample clustering and heatmap construction are given in Section "Materials and Methods." See Section "Materials and Methods" in the main text and Supplementary Figure $\mathbf{S} \mathbf{1}$ for the description of each treatment. The color scale is logarithmic to emphasize rare taxa, with $0.1 \%$ being set as the lower bound for logarithmic transformation. Numbers above the dendrogram indicate clusters described in the main text. Names of the OTUs were colored according to the three major modules in the Supplementary Figure S8, i.e., green, blue, and red corresponding to module 25,35 , and $45^{\circ} \mathrm{C}$, respectively (see Supplementary Figure S8). OTUs in black were not in the network.

two network modules did not clearly separate between the two moderate temperatures. Interestingly, there were only a few among these OTUs that were shared between all the different soils, i.e., Peptococcaceae OTU_5 and Heliobacteriaceae OTU_46 for the $45^{\circ} \mathrm{C}$ network module, and Geobacteraceae OTU_90 and Acidobacteria OTU_84 for the two moderate temperature network modules (Supplementary Figures S8-S10). There were also only few OTUs that were shared between two soils. The same was true for archaeal OTUs. Hence, most of the OTUs found in both network and heatmap were unique for each soil.

The assembly of OTUs from different functional groups into modules were largely different. For instance, OTUs 


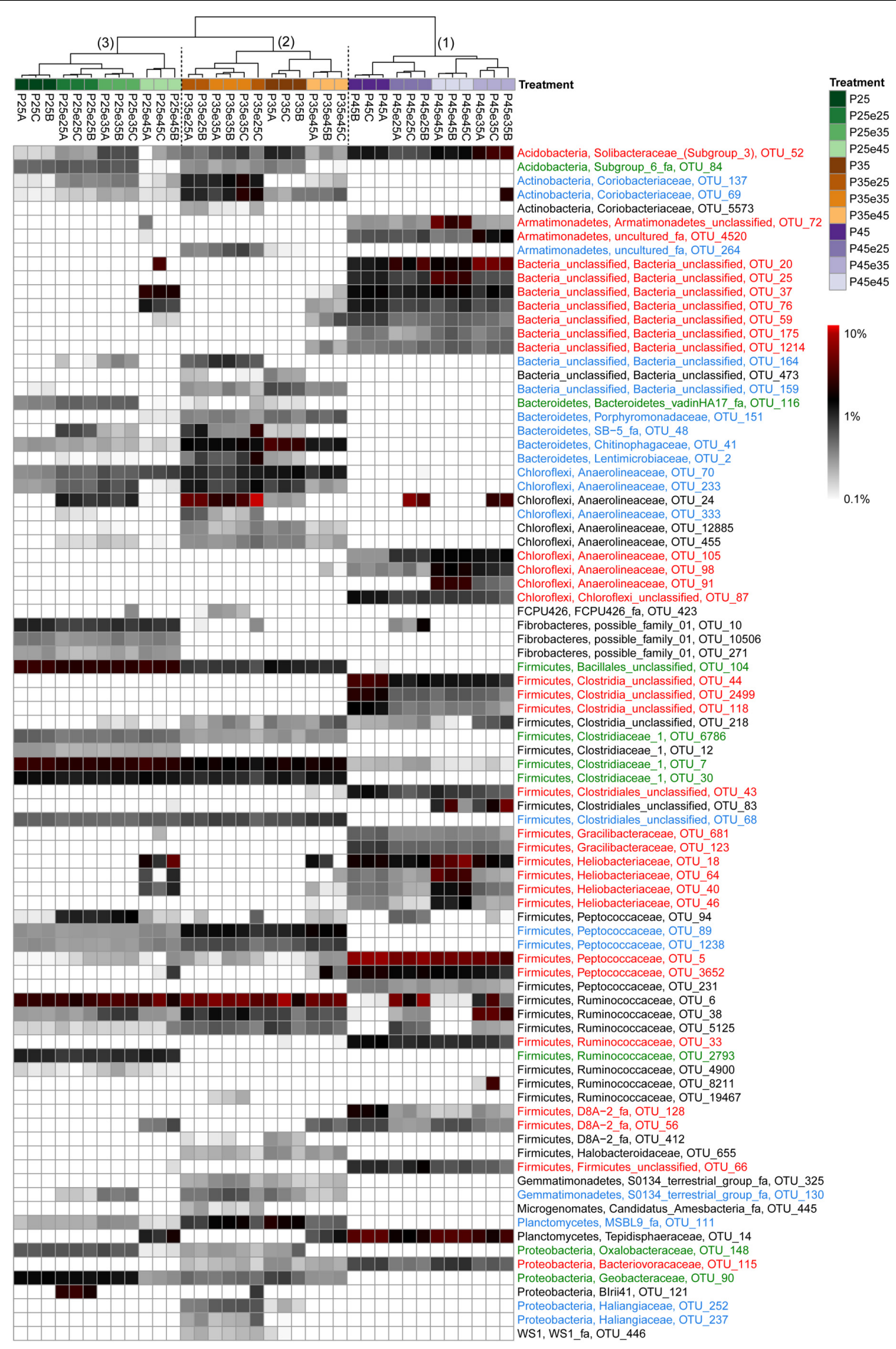

FIGURE 7| Relative abundances of selected bacterial OTUs that explain most of the differences between treatments in the Philippines paddy soils. Names of the OTUs were colored according to the three major modules in the Supplementary Figure S9, i.e., green, blue, and red corresponding to module 25,35 , and $45^{\circ} \mathrm{C}$, respectively (see Supplementary Figure S9). Refer to the legend of Figure 6 for additional details.

of hydrogenotrophic methanogens (Methanocellaceae and/or Methanobacteriaceae) occurred in network modules of either 45 or $35^{\circ} \mathrm{C}$ but not $25^{\circ} \mathrm{C}$ (Figure 5 and Supplementary Figures S8-S10), while OTUs of acetoclastic methanogens
(Methanosarcinaceae and/or Methanobacteriaceae) occurred only at modules of moderate temperatures (either 25 or $35^{\circ} \mathrm{C}$ ) (Figure 5 and Supplementary Figures S8-S10). There were also several archaeal OTUs, which did not belong to canonical 


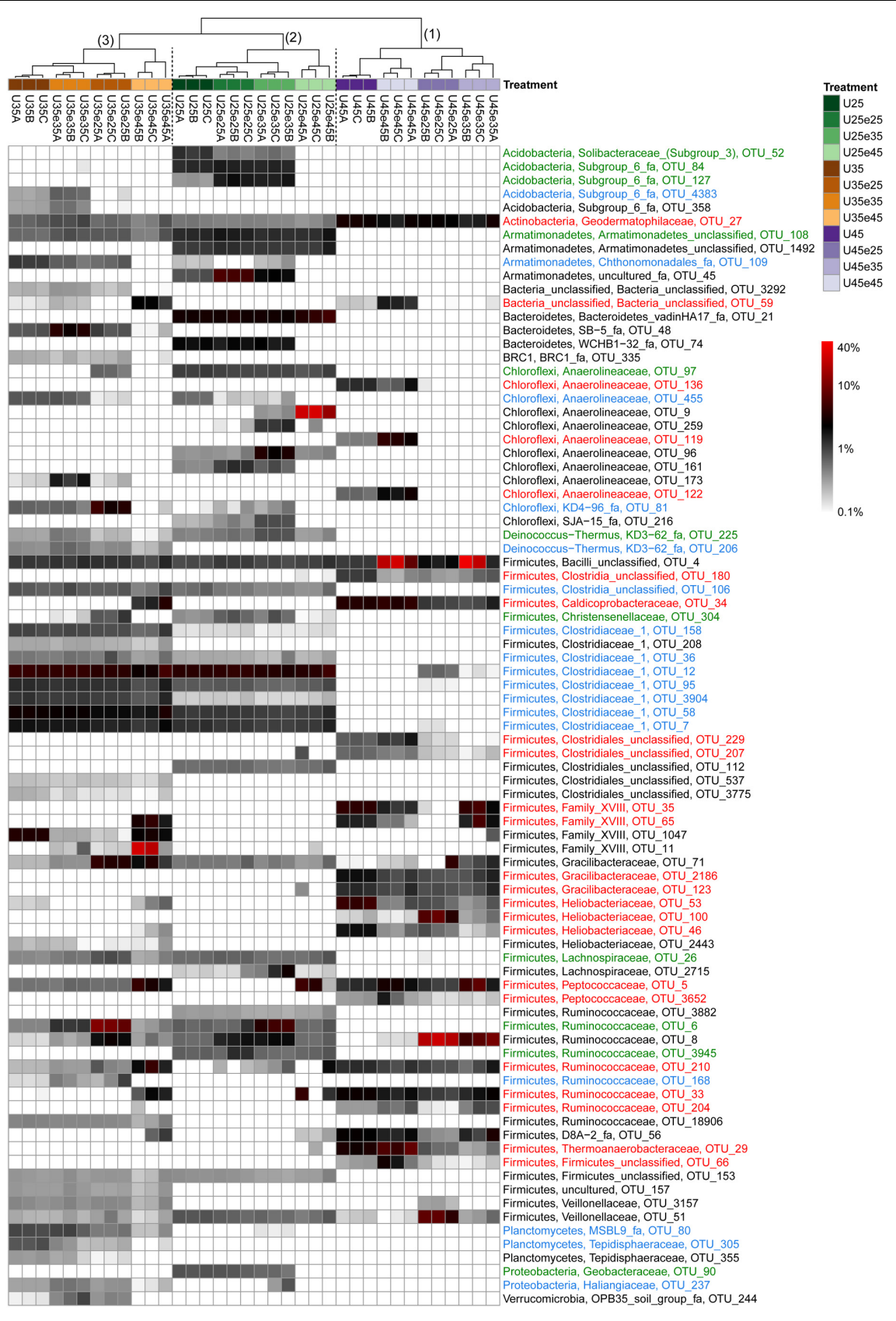

FIGURE 8 | Relative abundances of selected bacterial OTUs that explain most of the differences between treatments in Utah desert soil. Names of the OTUs were colored according to the three major modules in the Supplementary Figure S10, i.e., green, blue, and red corresponding to module 25,35 , and $45^{\circ} \mathrm{C}$, respectively (see Supplementary Figure S10). Refer to the legend of Figure $\mathbf{6}$ for additional details.

methanogens, but occurred in both heatmap and one of the network modules, i.e., Woesearchaeota (626 and 1382), Bathyarchaeota (138, 384, 398, and 1354), Thermoplasmata (277), and SCG $(77,135$, and 430$)$. They also preferentially occurred at moderate temperatures modules (Figures 6-8, Supplementary Figures S8-S10, and Supplementary Datasets 1-3).

In addition, some OTUs from the same functional group showed preferences for network modules and distinct temperature responses. For example, there were four major
Methanocellaceae OTUs $(13,31,287$, and 574) in Italian paddy soil which occurred in both heatmap and one of the network modules (Figure 5A and Supplementary Figure S8). One OTU (31) was exclusively found in soil that had experienced incubation at $45^{\circ} \mathrm{C}$, two OTUs (287 and 574) exclusively at $35^{\circ} \mathrm{C}$, and one OTU (13) in all treatments (Figure 5A). The same were true for some other methanogens (e.g., OTUs of Methanobacteriaceae) and bacteria from the same potential functional groups. Of all these bacterial OTUs, only those of 
Thermoanaerobacteraceae are clear candidates for the function as syntrophic acetate oxidizers (Liu and Conrad, 2010). Five Thermoanaerobacteraceae OTUs (55, 73, 29, 107, and 1930) occurred in Italian paddy soil only after experiencing incubation at $45^{\circ} \mathrm{C}$ (Figure 6). Although all five OTUs occurred in the same module, their responses to temperature were substantially different. The Philippines paddy soil, by contrast, did not contain a major Thermoanaerobacteraceae OTU (Figure 7), while the Utah desert soil only contained Thermoanaerobacteracea OTU_29 (Figure 8). Finally, the assembly into module and the temperature responses of the same OTU maybe differed between different soils. For example, Methanosarcinaceae OTU_42 showed the highest abundances at the two moderate temperatures and recovered when the temperature was changed back from $45^{\circ} \mathrm{C}$ to moderate temperatures in Italian soil. The relative abundances of OTU_42 were relatively stable in the Philippines soil (Figure 5B) while dramatically decreased in the Utah soil when pre-incubated at $45^{\circ} \mathrm{C}$ (Figure 5C). In addition, OTU_42 did not occur in the Italian soil network (Figure 5A and Supplementary Figure S8).

\section{DISCUSSION}

\section{Temperature Effects on the Soil Microbial Communities}

The incubation temperature had a decisive effect on the composition of the methanogenic microbial communities and their function in the different soils. The most important functional differences were the pathways of acetate conversion and methanogenesis in Italian and Utah soil, which were characterized by the operation of acetoclastic methanogenesis and of syntrophic acetate oxidation coupled to hydrogenotrophic methanogenesis at mesophilic $\left(25\right.$ and $\left.35^{\circ} \mathrm{C}\right)$ versus thermophilic $\left(45^{\circ} \mathrm{C}\right)$ incubation conditions, respectively. In the Philippines soil, by contrast, acetoclastic methanogenesis operated at both mesophilic and thermophilic conditions. These temperaturedependent functional differences, which are mainly seen from the different fractionation of ${ }^{13} \mathrm{C}$, have been discussed in detail in our previous publication (Liu et al., 2018). Our present study confirms and extends these observations with the results of factorial temperature shifts. Taken together, both studies allowed network analysis of the combined archaeal and bacterial microbial communities demonstrating that incubation temperature not only affected the function but also the composition of the methanogenic microbial communities. This analysis showed a distinct network module for each soil when incubated at $45^{\circ} \mathrm{C}$. There were two additional network modules for moderate temperatures, albeit not clearly separated with respect to $25^{\circ} \mathrm{C}$ versus $35^{\circ} \mathrm{C}$. However, in NMDS and heatmap analyses each single temperature treatment had a different effect. Nevertheless, the most drastic differences were between soils treated at moderate $\left(25\right.$ and $\left.35^{\circ} \mathrm{C}\right)$ versus elevated $\left(45^{\circ} \mathrm{C}\right)$ temperatures, as expected from previous experiments (Liu et al., 2018). However, even within these big clusters of community responses there was a distinct sub-clustering, sometimes distinguishing every single treatment. For example, shifts between incubations at $25^{\circ} \mathrm{C}$ versus $35^{\circ} \mathrm{C}$ often resulted in different compositions of the archaeal and the bacterial community. Interestingly, the temperature response of archaeal versus bacterial communities resulted in an almost identical pattern of clustering. By contrast, a temperature interval of only $5^{\circ} \mathrm{C}$ in wetland soil from the Tibetan Plateau showed no effect on pathway and community composition of methanogenic archaea (Cui et al., 2015). Our results show that temperature at sufficiently large intervals is a very important regulator of community composition (Conrad, 2008; Roussel et al., 2015). They also show that there was a substantial taxonomic richness within the different functional groups (e.g., hydrogenotrophic methanogenesis; see discussion below) indicating that temperature is an important environmental effector causing the emergence of functional redundancy (Louca et al., 2018).

The incubation temperature also affected the size of the microbial populations, but only to a relatively small extent. The archaeal and bacterial counts (given as 16S rRNA gene copy numbers) always were in a reasonable range of $10^{8}$ to $10^{10}$ per gram soil with Bacteria usually being by a factor of 10 more numerous than archaea. Higher bacterial than of archaeal counts have commonly been observed (Fernandez Scavino et al., 2013; Breidenbach et al., 2017; Reim et al., 2017). Numbers apparently increased from the pre-incubation to the subsequent incubation indicating growth of microbial populations. This observation is reasonable, since the soil had been amended with cellulose as additional substrate to equalize substrate availability across the different soils. As microbial growth occurred at the different temperatures, the individual bacterial and archaeal populations were apparently affected to different extents, thus resulting in different community compositions.

Temperature also affected the overall diversity of the microbial communities. In particular, diversity in Italian paddy soil and Utah desert soil was generally lower when the soil had been incubated at $45^{\circ} \mathrm{C}$ compared to soil incubated at moderate temperatures only. Such decrease of diversity was found for both Archaea and Bacteria, which was not consistently reversible, when the temperature was changed back from $45^{\circ} \mathrm{C}$ to moderate conditions. We assume that the time for recovery was not sufficient, since microbial counts were not consistently higher when temperature was shifted back from $45^{\circ} \mathrm{C}$ to moderate conditions. An earlier study with Italian paddy soil indicated that recovery might be possible when the soil is in addition inoculated with soil from moderate temperatures (Noll et al., 2010). Therefore, since we did not use inoculation in our experiments, we assume that recovery was not possible since the microbes had been killed by exposure to $45^{\circ} \mathrm{C}$. In the Philippines paddy soil, decrease of diversity was observed for Bacteria, while archaeal diversity was not so much affected.

\section{Temperature Modulation of Important Microbial OTUs}

Our study demonstrates the modulation of the microbial community composition by temperature in three different soils. This intrinsic ability of the soil methanogenic microbial 
communities is remarkable. Although elevated temperatures, also well beyond $45^{\circ} \mathrm{C}$, do occur in tropical soils, they are probably not reached during the time when the soils are flooded and the methanogenic communities are active. The fact, that the soils nevertheless possess populations of moderately thermophilic Bacteria and Archaea indicates that the non-flooded conditions might also affect particular microbial populations.

It is difficult to deduce the importance of microbial species for specific functions, since these are not or not exactly known for most of the detected OTUs. For methanogenic microbial communities, however, some connection can be made, since we are relatively sure about the physiological capacities of many methanogenic archaeal taxa. Our study revealed among acetoclastic (especially Methanotrichaceae) and hydrogenotrophic (especially Methanocellaceae) methanogenic archaea quite some redundancy with respect to different taxa (OTUs), some of them responding differently upon temperature change, others perhaps contributing to functional redundancy (Louca et al., 2018).

Acetoclastic methanogenesis is usually the process that is responsible for acetate consumption during the methanogenic degradation of organic matter. In the different soils, acetoclastic methanogenesis (as identified by relatively low $\alpha_{\text {app }}$ values) only operated when putative acetoclastic archaeal taxa (Methanosarcinaceae, Methanotrichaceae) were present. The Methanotrichaceae in Italian paddy soil were strictly mesophilic and could only serve the acetoclastic function at moderate temperatures. This result is consistent with previous experiments, albeit they did not extend to the OTU level (Conrad et al., 2009). The Methanotrichaceae were represented by 8 different OTUs with relative abundance larger than $0.8 \%$, thus presenting a substantial functional redundancy. Many of the OTUs were organized in one of the network modules indicating that their functioning as acetoclastic methanogens may be affected by interactions with other microbes. The acetoclastic archaeal community in Italian paddy soil consisted in addition of one major Methanosarcinaceae OTU_42. Similarly to the Methanotrichaceae this OTU was missing whenever the soil was incubated at $45^{\circ} \mathrm{C}$, but recovered when the incubation temperature was again decreased to moderate conditions. The recovery of Methanosarcinaceae OTU_42 after temperature shift from 45 to $25^{\circ} \mathrm{C}$ or $35^{\circ} \mathrm{C}$ indeed resulted in a gradual recovery of the acetoclastic function as seen by the decrease of $\alpha_{\text {app }}$ values (Figure 2C). The shape of the $\alpha_{\text {app }}$ curves possibly indicates the temporal recovery of the acetoclastic Methanosarcina, which happened earlier at $35^{\circ} \mathrm{C}$ than at $25^{\circ} \mathrm{C}$ (Figure 2C).

The acetoclastic archaeal community in the Philippines paddy soil consisted of one major Methanotrichaceae OTU_205 and one Methanosarcinaceae OTU_42, which both occurred also in the Italian paddy soil. Hence there was little functional redundancy in this soil. It is noteworthy that these two acetoclastic methanogens were thermotolerant in the Philippines soil, but were not thermotolerant in the Italian soil. We conclude that Methanotrichaceae OTU_205 and Methanosarcinaceae
OTU_42 exist as taxonomically closely related thermotolerant and thermointolerant ecotype. The existence of such ecotypes is commonly found in the literature (Simankova et al., 2003; Acinas et al., 2004; Wu et al., 2006; Becraft et al., 2011). Interestingly, the Utah desert soil also contained Methanosarcinaceae OTU_42, but like the Italian soil only as thermointolerant ecotype. This OTU was the sole acetoclastic methanogen in Utah soil, which thus showed no functional redundancy. Although it occurred at moderate temperatures, its population strongly decreased after pre-incubation at $45^{\circ} \mathrm{C}$ (Figure 5C), so that acetate was no longer consumed and accumulated (Supplementary Table S2).

The major hydrogenotrophic taxa in Italian paddy soil were Methanobacteriaceae and Methanocellaceae. Methanosarcinaceae can also use $\mathrm{H}_{2} / \mathrm{CO}_{2}$ as substrate, albeit not as efficiently as Methanobacteriaceae and Methanocellaceae (Thauer et al., 2008). Among the Methanocellaceae there was one OTU (31), which was exclusively found at $45^{\circ} \mathrm{C}$, while another OTU (13) was found at all temperatures. In the Philippines paddy soil, however, this OTU (13) only occurred, when the soil had not been pre-incubated at $45^{\circ} \mathrm{C}$, again indicating the existence of ecotypes. In Utah desert soil, there were two Methanocellaceae OTUs (287, 3964), but not at elevated temperatures. Only one Methanocellales OTU_50 also occurred at $45^{\circ} \mathrm{C}$. However, the more important hydrogenotrophic methanogenic taxon at $45^{\circ} \mathrm{C}$ was Methanobacteriaceae OTU_3, which was also found in Italian paddy soil. A second Methanobacteriaceae OTU_1034, which was also found in Italian and the Philippines paddy soils, was less abundant and seemed to prefer moderate temperatures. Hence, hydrogenotrophic methanogens were functionally redundant.

Bathyarchaeota were found in all three soils with 2-3 different OTUs. Bathyarchaeota, which belong to the TACK superphylum (Adam et al., 2017; Spang et al., 2017) have not yet been cultured. Although metagenomic data suggest that they may be potential methanogens (Evans et al., 2015; Spang et al., 2017), other physiological functions are also conceivable (He et al., 2016; Spang et al., 2017; Xiang et al., 2017). One OTU (138) occurred in all three soils, but only at moderate temperatures. Potentially thermophilic OTUs only occurred in the Philippines paddy soil, with unknown function. Woesearchaeota, which belong to the DPANN superphylum, were found in the Italian and Philippines paddy soils at moderate temperatures. However, their functions are unknown, albeit probably not methanogenic (Adam et al., 2017; Spang et al., 2017). The SCG archaea, which occurred as several OTUs in all the soils at all temperatures most probably did also not function as methanogens (Lu et al., 2017).

If acetoclastic methanogenesis does not operate in methanogenic systems, acetate consumption can only be achieved by acetate-oxidizing bacteria that are syntrophically coupled to hydrogenotrophic methanogens. We identified the operation of syntrophic acetate oxidation by rather high $\alpha_{\text {app }}$ values and the fact that acetate did not accumulate. Such conditions happened in Italian paddy soil, when the temperature was increased to $45^{\circ} \mathrm{C}$ as reported before (Conrad et al., 2009). Syntrophic Thermoanaerobacteraceae have been identified 
as being responsible for acetate consumption under these conditions (Liu and Conrad, 2010). Thermoanaerobacteraceae were also found in our experiments. The relative abundance of this taxon (represented by 4 major OTUs) specifically increased whenever the soil was incubated at $45^{\circ} \mathrm{C}$. The Philippines paddy soil, which displayed acetoclastic methanogenesis at all the different temperatures, did not contain a major Thermoanaerobacteraceae OTU. However, the Utah desert soil contained Thermoanaerobacteracea OTU_29, which was identical to the OTU identified in Italian paddy soil. The failed instantaneous replacement of acetoclastic methanogenesis in Utah soil by syntrophic acetate oxidizers resulted in Utah soil in decrease of methanogenic activity and accumulation of acetate.

Besides the putatively acetate-oxidizing Thermoanaerobacteraceae temperature effects were also found for other Bacteria. The functions of these different taxa within the methanogenic degradation process are unclear, but most probably involve various hydrolytic and fermentation processes. Helicobacteraceae, for example, may be involved in anaerobic oxidation of propionate, possibly even acetate (Noll et al., 2010; Sattley and Madigan, 2014; Liu et al., 2018; Peng et al., 2018). The different bacterial taxa were separated together with archaeal taxa into three major network modules, the most distinct being those in soils exposed to $45^{\circ} \mathrm{C}$ versus those exposed to moderate temperatures (either 25 or $35^{\circ} \mathrm{C}$ ). Whereas elevated temperature $\left(45^{\circ} \mathrm{C}\right)$ seemed to favor Heliobacteriaceae OTUs in all three soils, and Peptococcaceae OTUs in particular in the Philippines soil, moderate temperatures seemed to generally favor Anaerolineaceae and Ruminococcaceae OTUs. There were only four bacterial OTUs that were shared between all of the soils, two occurring in the network modules at $45^{\circ} \mathrm{C}$ and two in those at 25 or $35^{\circ} \mathrm{C}$. These OTUs belonged to Heliobacteriaceae, Peptococcaceae, Geobacteraceae, and Acidobacteria, respectively. Besides this core of bacterial taxa, each soil possessed its own characteristic bacterial community. There were numerous bacterial taxa that were affected by temperature specifically in each soil. In general, soils apparently had their individual microbial communities, which reacted to temperature exposure.

\section{CONCLUSION}

Our results demonstrated quite some taxonomic redundancy of the functional microbial groups, in particular syntrophic acetate oxidation and acetoclastic and hydrogenotrophic methanogenesis, after exposure to mesophilic $\left(25\right.$ and $\left.35^{\circ} \mathrm{C}\right)$ and thermophilic $\left(45^{\circ} \mathrm{C}\right)$ temperatures. Our results also show that the methanogenic bacterial and archaeal communities were indeed organized in co-occurrence networks in the three different soils studied. This observation indicates that methanogenic soil microbial communities are organized in distinct temperaturedependent modules rather than in a random way.

Our results are consistent with our initial hypothesis that function is determined by the bacterial and/or archaeal community structures. The pathways of $\mathrm{CH}_{4}$ production changed between acetoclastic methanogenesis and syntrophic acetate oxidation, always combined with hydrogenotrophic methanogenesis. These changes were accompanied by shifts in community composition. These shifts were in particular seen by changes in the relative abundance of particular OTUs belonging to hydrogenotrophic and acetoclastic methanogenic taxa and to potentially syntrophic acetateoxidizing Thermoanaerobacteraceae. However, there were also changes in other bacterial taxa that were probably involved in hydrolytic and fermentation processes.

Our results also supported our second hypothesis that temperature affects the taxonomic structure within the different functional groups. There was a substantial taxonomic redundancy allowing for the different functions within the methanogenic pathways (Louca et al., 2018). We also realized an amazing individuality of the different soils with respect to microbial OTUs, which were hardly shared. Even within shared OTUs we realized different adaptabilities to temperature change suggesting the existence of ecotypes within the same OTU.

Thirdly, we realized that the overall microbial diversity was reduced at $45^{\circ} \mathrm{C}$. Once the diversity was lost, it was not fully regenerated by reversal of incubation temperatures, probably since the microbial seedbank was no longer available due to laboratory confinement. In summary, our experiments showed that temperature was an important regulator for structure and function of methanogenic microbial communities, which in all the soils tested displayed distinct temperature modules within co-occurrence networks.

Nevertheless, taxonomic microbial community structures in each soil were different and reacted individually to temperature treatment for reasons that are presently not understood. In particular it is unclear, which factors allow soils possessing or not possessing thermophilic populations of acetoclastic methanogens. The mechanistic basis for why structure and function of the methanogenic microbial communities drastically changed at around $45^{\circ} \mathrm{C}$, and not at 25 or $35^{\circ} \mathrm{C}$, is also unknown. We think that future research should consider the observation that acetate consumption by the archaeal acetoclastic methanogenic pathway is replaced by the bacterial WoodLjungdahl pathway. It should also consider the fact that Bacteria and Archaea possess different membrane lipids with inherently different functional constraints (Valentine, 2007).

\section{DATA AVAILABILITY}

The datasets generated for this study can be found in NCBI Sequence Read Archive, SRP133538.

\section{AUTHOR CONTRIBUTIONS}

PL performed the data analysis. MK carried out the experiments. $\mathrm{PL}$ and RC wrote the manuscript.

\section{FUNDING}

This study was financially supported by the Fonds der Chemischen Industrie. 


\section{ACKNOWLEDGMENTS}

We thank Prof. J. C. Ensign for sampling soil in Utah and P. Claus for help in analysis of ${ }^{13} \mathrm{C}$. We thank the two reviewers for constructive comments and suggestions.

\section{REFERENCES}

Acinas, S. G., Klepac-Ceraj, V., Hunt, D. E., Pharino, C., Ceraj, I., Distel, D. L., et al. (2004). Fine-scale phylogenetic architecture of a complex bacterial community. Nature 430, 551-554. doi: 10.1038/nature02649

Adam, P. S., Borrel, G., Brochier-Armanet, C., and Gribaldo, S. (2017). The growing tree of Archaea: new perspectives on their diversity, evolution and ecology [review]. ISME J. 11, 2407-2425. doi: 10.1038/ismej.2017.122

Angel, R., Claus, P., and Conrad, R. (2012). Methanogenic archaea are globally ubiquitous in aerated soils and become active under wet anoxic conditions. ISME J. 6, 847-862. doi: 10.1038/ismej.2011.141

Asakawa, S., and Kimura, M. (2008). Comparison of bacterial community structures at main habitats in paddy field ecosystem based on DGGE analysis [review]. Soil Biol. Biochem. 40, 1322-1329. doi: 10.1016/j.soilbio.2007.09.024

Bates, S. T., Berg-Lyons, D., Caporaso, J. G., Walters, W. A., Knight, R., and Fierer, N. (2011). Examining the global distribution of dominant archaeal populations in soil. ISME J. 5, 908-917. doi: 10.1038/ismej.2010.171

Becraft, E. D., Cohan, F. M., Kühl, M., Jensen, S. I., and Ward, D. M. (2011). Fine-scale distribution patterns of Synechococcus ecological diversity in microbial mats of Mushroom Spring, Yellowstone National Park. Appl. Environ. Microbiol. 77, 7689-7697. doi: 10.1128/AEM.05927-11

Blondel, V. D., Guillaume, J. L., Lambiotte, R., and Lefebvre, E. (2008). Fast unfolding of communities in large networks. J. Stat. Mech. Theory Exp. 2008:P10008. doi: 10.1088/1742-5468/2008/10/P10008

Breidenbach, B., Brenzinger, K., Brandt, F. B., Blaser, M. B., and Conrad, R. (2017). The effect of crop rotation between wetland rice and upland maize on the microbial communities associated with roots. Plant Soil 419, 435-445. doi: 10.1007/s11104-017-3351-5

Caporaso, J. G., Kuczynski, J., Stombaugh, J., Bittinger, K., Bushman, F. D., Costello, E. K., et al. (2010). QIIME allows analysis of highthroughput community sequencing data. Nat. Methods 7, 335-336. doi: $10.1038 /$ nmeth.f.303

Conrad, R. (2005). Quantification of methanogenic pathways using stable carbon isotopic signatures: a review and a proposal. Org. Geochem. 36, 739-752. doi: 10.1016/j.orggeochem.2004.09.006

Conrad, R. (2007). Microbial ecology of methanogens and methanotrophs. Adv. Agron. 96, 1-63. doi: 10.1016/S0065-2113(07)96005-8

Conrad, R. (2008). “Temperature effects on methanogenic microbial communities," in Microbes and the Environment: Perspectives and Challenges, eds S. J. Liu and H. L. Drake (Beijing: Science Press), 35-40.

Conrad, R., Klose, M., Lu, Y., and Chidthaisong, A. (2012). Methanogenic pathway and archaeal communities in three different anoxic soils amended with rice straw and maize straw. Front. Microbiol. 3:4. doi: 10.3389/fmicb.2012.00004

Conrad, R., Klose, M., and Noll, M. (2009). Functional and structural response of the methanogenic microbial community in rice field soil to temperature change. Environ. Microbiol. 11, 1844-1853. doi: 10.1111/j.1462-2920.2009.01 909.x

Cui, M., Ma, A., Qi, H., Zhuang, X., Zhuang, G., and Zhao, G. (2015). Warmer temperature accelerates methane emissions from the Zoige wetland on the Tibetan Plateau without changing methanogenic community composition. Sci. Rep. 5:11616. doi: 10.1038/srep11616

Edgar, R. C. (2010). Search and clustering orders of magnitude faster than BLAST. Bioinformatics 26, 2460-2461. doi: 10.1093/bioinformatics/btq461

Edgar, R. C. (2013). UPARSE: highly accurate OTU sequences from microbial amplicon reads. Nat. Methods 10, 996-998. doi: 10.1038/nmeth.2604

Evans, P. N., Parks, D. H., Chadwick, G. L., Robbins, S. J., Orphan, V. J., Golding, S. D., et al. (2015). Methane metabolism in the archaeal phylum Bathyarchaeota revealed by genome-centric metagenomics. Science 350, 434-438. doi: 10.1126/ science.aac 7745

\section{SUPPLEMENTARY MATERIAL}

The Supplementary Material for this article can be found online at: https://www.frontiersin.org/articles/10.3389/fmicb. 2019.00496/full\#supplementary-material

Fernandez Scavino, A., Ji, Y., Pump, J., Klose, M., Claus, P., and Conrad, R. (2013). Structure and function of the methanogenic microbial communities in Uruguayan soils shifted between pasture and irrigated rice fields. Environ. Microbiol. 15, 2588-2602. doi: 10.1111/1462-2920.12161

Fey, A., Chin, K. J., and Conrad, R. (2001). Thermophilic methanogens in rice field soil. Environ. Microbiol. 3, 295-303. doi: 10.1046/j.1462-2920.2001.00195.x

Fey, A., Claus, P., and Conrad, R. (2004). Temporal change of 13C-isotope signatures and methanogenic pathways in rice field soil incubated anoxically at different temperatures. Geochim. Cosmochim. Acta 68, 293-306. doi: 10.1016/ S0016-7037(03)00426-5

Fruchterman, T. M., and Reingold, E. M. (1991). Graph drawing by force-directed placement. J. Softw. Pract. Exper. 21, 1129-1164. doi: 10.1002/spe.4380211102

Fu, B., Conrad, R., and Blaser, M. (2018). Potential contribution of acetogenesis to anaerobic degradation in methanogenic rice field soils. Soil Biol. Biochem. 119, 1-10. doi: 10.1016/j.soilbio.2017.10.034

He, Y., Li, M., Perumal, V., Feng, X., Fang, J., Xie, J., et al. (2016). Genomic and enzymatic evidence for acetogenesis among multiple lineages of the archaeal phylum Bathyarchaeota widespread in marine sediments. Nat. Microbiol. 1:16035. doi: 10.1038/NMICROBIOL.2016.35

Hernandez, M., Conrad, R., Klose, M., Ma, K., and Lu, Y. H. (2017). Structure and function of methanogenic microbial communities in soils from flooded rice and upland soybean fields from Sanjiang plain, NE China. Soil Biol. Biochem. 105, 81-91. doi: 10.1016/j.soilbio.2016.11.010

Liu, F. H., and Conrad, R. (2010). Thermoanaerobacteriaceae oxidize acetate in methanogenic rice field soil at 50oC. Environ. Microbiol. 12, 2341-2354. doi: 10.1111/j.1462-2920.2010.02289.x

Liu, P., Klose, M., and Conrad, R. (2018). Temperature effects on structure and function of the methanogenic microbial communities in two paddy soils and one desert soil. Soil Biol. Biochem. 124, 236-244. doi: 10.1016/j.soilbio.2018.06.024

Louca, S., Polz, M. F., Mazel, F., Albright, M. B. N., Huber, J. A., O’Connor, M. I., et al. (2018). Function and functional redundancy in microbial systems [review]. Nat. Ecol. Evol. 2, 936-943. doi: 10.1038/s41559-018-0519-1

Lu, X. D., Seuradge, B. J., and Neufeld, J. D. (2017). Biogeography of soil Thaumarchaeota in relation to soil depth and land usage. FEMS Microbiol. Ecol. 93:fiw246. doi: 10.1093/femsec/fiw246

Lu, Y., Fu, L., Lu, Y., Hugenholtz, F., and Ma, K. (2015). Effect of temperature on the structure and activity of a methanogenic archaeal community during rice straw decomposition. Soil Biol. Biochem. 81, 17-27. doi: 10.1016/j.soilbio.2014. 10.031

Martin, M. (2011). Cutadapt removes adapter sequences from high-throughput sequencing reads. EMBnet J. 17, 10-12. doi: 10.14806/ej.17.1.200

McMurdie, P. J., and Holmes, S. (2013). phyloseq: an R package for reproducible interactive analysis and graphics of microbiome census data. PLoS One 8:e61217. doi: 10.1371/journal.pone.0061217

Mendiburu, F., and Simon, R. (2015). Agricolae - Ten years of an open source statistical tool for experiments in breeding, agriculture and biology. PeerJ PrePrints 3:e1404v1. doi: 10.7287/peerj.preprints.1404v1

Noll, M., Klose, M., and Conrad, R. (2010). Effect of temperature change on the composition of the bacterial and archaeal community potentially involved in the turnover of acetate and propionate in methanogenic rice field soil. FEMS Microbiol. Ecol. 73, 215-225. doi: 10.1111/j.1574-6941.2010.00883.x

Oksanen, J., Kindt, R., Legendre, P., O’Hara, B., Stevens, M. H. H., Oksanen, M. J., et al. (2007). The vegan package. Community Ecol. Package 10, 631-637.

Oren, A. (2014). "The family Methanotrichaceae," in The Prokaryotes, eds E. Rosenberg, E. F. DeLong, S. Lory, E. Stackebrandt, and F. Thompson (Berlin: Springer), 298-306

Peng, J., Wegner, C. E., Bei, Q., Liu, P., and Liesack, W. (2018). Metatranscriptomics reveals a differential temperature effect on the structural 
and functional organization of the anaerobic food web in rice field soil. Microbiome 6:169. doi: 10.1186/s40168-018-0546-9

Pruesse, E., Quast, C., Knittel, K., Fuchs, B. M., Ludwig, W., Peplies, J., et al. (2007). SILVA: a comprehensive online resource for quality checked and aligned ribosomal RNA sequence data compatible with ARB. Nucleic Acids Res. 35, 7188-7196. doi: 10.1093/nar/gkm864

Reim, A., Hernandez, M., Klose, M., Chidthaisong, A., Yuttiham, M., and Conrad, R. (2017). Response of methanogenic microbial communities to desiccation stress in flooded and rain-fed paddy soil from Thailand. Front. Microbiol. 8:785. doi: 10.3389/fmicb.2017.00785

Roussel, E. G., Cragg, B. A., Webster, G., Sass, H., Tang, X., Williams, A. S., et al. (2015). Complex coupled metabolic and prokaryotic community responses to increasing temperatures in anaerobic marine sediments: critical temperatures and substrate changes. FEMS Microb. Ecol. 91:fiv084. doi: 10.1093/femsec/ fiv084

Rui, J., Peng, J., and Lu, Y. (2009). Succession of bacterial populations during plant residue decomposition in rice field soil. Appl. Environ. Microbiol. 75, 4879-4886. doi: 10.1128/AEM.00702-09

Rui, J., Qiu, Q., and Lu, Y. (2011). Syntrophic acetate oxidation under thermophilic methanogenic condition in Chinese paddy field soil. FEMS Microbiol. Ecol. 77, 264-273. doi: 10.1111/j.1574-6941.2011.01 104.X

Sattley, W. M., and Madigan, M. T. (2014). "The family Heliobacteriaceae," in The Prokaryotes, eds E. Rosenberg, E. F. DeLong, S. Lory, E. Stackebrandt, and F. Thompson (Berlin: Springer), 185-196.

Schink, B., and Stams, A. J. M. (2013). "Syntrophism among prokaryotes”, in The Prokaryotes: Prokaryotic Communities and Ecophysiology, eds E. Rosenberg, E. F. DeLong, S. Lory, E. Stackebrandt, and F. Thompson (Berlin: Springer), 471-493.

Simankova, M. V., Kotsyurbenko, O. R., Lueders, T., Nozhevnikova, A. N., Wagner, B., Conrad, R., et al. (2003). Isolation and characterization of new strains of methanogens from cold terrestrial habitats. Syst. Appl. Microbiol. 26, 312-318. doi: 10.1078/072320203322346173

Spang, A., Caceres, E. F., and Ettema, T. J. G. (2017). Genomic exploration of the diversity, ecology, and evolution of the archaeal domain of life [review]. Science 357:eaaf3883. doi: 10.1126/science.aaf3883

Thauer, R. K., Kaster, A. K., Seedorf, H., Buckel, W., and Hedderich, R. (2008). Methanogenic archaea: ecologically relevant differences in energy conservation. Nat. Rev. Microbiol. 6, 579-591. doi: 10.1038/nrmicro1931

Valentine, D. L. (2007). Adaptations to energy stress dictate the ecology and evolution of the Archaea [Review]. Nat. Rev. Microbiol. 5, 316-323. doi: 10. 1038/nrmicro1619

Wang, Q., Garrity, G. M., Tiedje, J. M., and Cole, J. R. (2007). Naive Bayesian classifier for rapid assignment of rRNA sequences into the new bacterial taxonomy. Appl. Environ. Microbiol. 73, 5261-5267. doi: 10.1128/AEM.00062-07

Wegner, C. E., and Liesack, W. (2016). Microbial community dynamics during the early stages of plant polymer breakdown in paddy soil. Environ. Microbiol. 18, 2825-2842. doi: 10.1111/1462-2920.12815

Whiticar, M. J. (1999). Carbon and hydrogen isotope systematics of bacterial formation and oxidation of methane. Chem. Geol. 161, 291-314. doi: 10.1016/ S0009-2541(99)00092-3

Wiegel, J. (1990). Temperature spans for growth: hypothesis and discussion. FEMS Microbiol. Rev. 75, 155-170. doi: 10.1111/j.1574-6968.1990.tb04092.x

Williams, R. J., Howe, A., and Hofmockel, K. (2014). Demonstrating microbial cooccurrence pattern analyses within and between ecosystems. Front. Microbiol. 5:358. doi: 10.3389/fmicb.2014.00358

Wu, X. L., Friedrich, M. W., and Conrad, R. (2006). Diversity and ubiquity of thermophilic methanogenic archaea in temperate anoxic soils. Environ. Microbiol. 8, 394-404. doi: 10.1111/j.1462-2920.2005.00904.X

Xiang, X., Wang, R., Wang, H., Gong, L., Man, B., and Xu, Y. (2017). Distribution of Bathyarchaeota communities across different terrestrial settings and their potential ecological functions. Sci. Rep. 7:45028. doi: 10.1038/srep45028

Yao, H., and Conrad, R. (2000). Effect of temperature on reduction of iron and production of carbon dioxide and methane in anoxic wetland rice soils. Biol. Fertil. Soils 32, 135-141. doi: 10.1007/s003740000227

Yao, H., Conrad, R., Wassmann, R., and Neue, H. U. (1999). Effect of soil characteristics on sequential reduction and methane production in sixteen rice paddy soils from China, the Philippines, and Italy. Biogeochemistry 47, 269-295. doi: 10.1007/BF00992910

Yvon-Durocher, G., Allen, A. P., Bastviken, D., Conrad, R., Gudasz, C., St-Pierre, A., et al. (2014). Methane fluxes show consistent temperature dependence across microbial to ecosystem scales. Nature 507, 488-491. doi: $10.1038 /$ nature 13164

Zinder, S. H. (1993). "Physiological ecology of methanogens," in Methanogenesis: Ecology, Physiology, Biochemistry and Genetics, ed. J. G. Ferry (New York, NY: Chapman \& Hall), 128-206.

Conflict of Interest Statement: The authors declare that the research was conducted in the absence of any commercial or financial relationships that could be construed as a potential conflict of interest.

Copyright (c) 2019 Liu, Klose and Conrad. This is an open-access article distributed under the terms of the Creative Commons Attribution License (CC BY). The use, distribution or reproduction in other forums is permitted, provided the original author(s) and the copyright owner(s) are credited and that the original publication in this journal is cited, in accordance with accepted academic practice. No use, distribution or reproduction is permitted which does not comply with these terms. 